\title{
A Multiplexed Chemical Sensing CMOS Imager
}

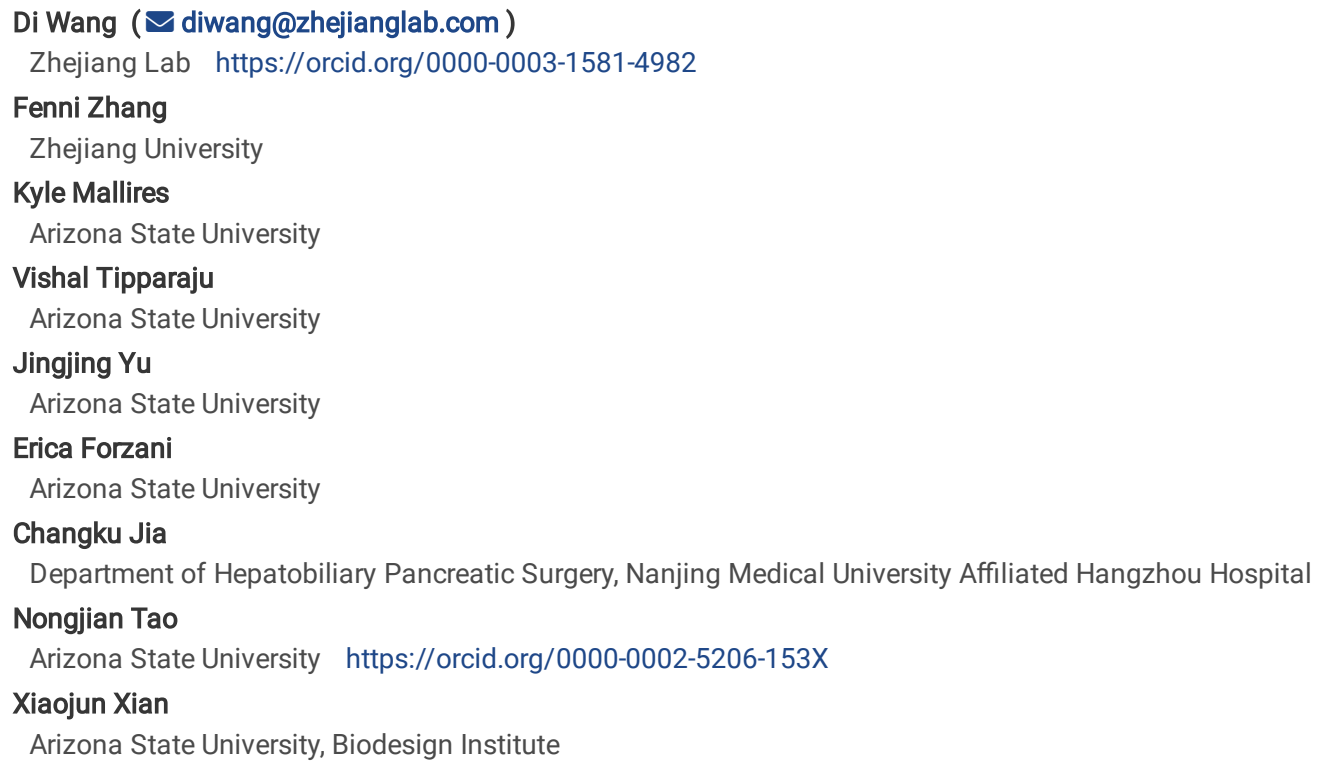




\section{Abstract}

A miniaturized and multiplexed chemical sensing technology is urgently needed to empower mobile devices, Internet-of-Things (loTs) and robots for various new applications. Here, we show that a complementary metal-oxide-semiconductor (CMOS) imager can be turned into a multiplexed colorimetric sensing chip by coating micron-scale colorimetric sensing spots on the imager surface. Each sensing spot contains chemical sensing materials and nanoparticles for colorimetric signal enhancement. The sensitivity is spot-size invariant, and high-performance chemical sensing can be achieved on sensing spot as small as $\sim 10 \mu \mathrm{m}$. This great scalability combined with millions of pixels of a CMOS imager offers a promising platform for highly integrated chemical sensors. Moreover, the chemical CMOS chip can be readily integrated with mobile electronics. As a proof-of-concept, we have built a smartphone accessary based on this chemical CMOS chip for personal health management. We anticipate that this new platform will pave the way for the widespread application of chemical sensing, such as mobile health (mHealth), loTs, electronic nose, and smart homes.

\section{Introduction}

Modern computer and electronic technologies, including artificial intelligence, are becoming ever more powerful for information processing. There is a lack of proportional progress in information collection, which is performed by various sensors ${ }^{1-3}$. Sensors are an integral part of today's electronics. For example, a smartphone is equipped with more than a dozen sensors, including magnetic sensor, accelerometer, gyroscope, microphone, thermometer, proximity sensor, barometer, and complementary metal-oxide-semiconductor (CMOS) imagers ${ }^{4,5}$. However, all these sensors measure physical quantities, and none can sense chemicals. A miniaturized and multiplexed chemical sensor would empower mobile devices to detect early signs of diseases, alert contamination of food and drinking water, and sense danger of toxic chemicals in air ${ }^{6-8}$.

Low cost and miniaturized chemical sensors have been actively pursued ${ }^{9,10}$. A promising example is metal oxide sensors, which are sensitive, miniaturized and compatible with electronics. However, they lack selectivity, and the high power consumption limits their scalability to meet the need of integrating increasing number of sensors ${ }^{11,12}$. Colorimetric sensors detect a color change originated from the specific reaction of a target analyte with a sensing material ${ }^{13,14}$. The most successful example of colorimetric gas sensing is the detection tubes, each containing a sensing material sealed in a glass tube (Fig. 1a). Breaking the tube exposes the sensing material to a chemical and leads to a color change. Millions of detection tubes are being sold each year for safeguarding workers in chemical and related plants, firefighters on duty, and preventing air pollution and chemical leakage ${ }^{15,16}$. While useful, these tubes are bulky, time-consuming, semi-quantitative, and each detects typically only one analyte ${ }^{17-19}$. Alternatively, a colorimetric sensing array can be printed on a substrate and then imaged with an optical system ${ }^{20-23}$ (Fig. 1d). This approach is, however, not compatible with integrated circuits, and difficult to miniaturize because of the large size of each printed sensing element and use of optical components.

CMOS imager is an attractive platform for multiplexed optoelectronic sensing. A today's CMOS imager with a size of a few millimeters offers millions of pixels, each as a low noise optical sensor, yet it is fully compatible with modern electronics and widely used in every smartphone, tablet, personal computer and security camera. This fast-growing demand for CMOS imagers has driven their price down to a few dollars, which allows CMOS imagers to be used even as disposable sensors. Previously we have demonstrated sub-ppm level ammonia detection can be achieved on the liquid phase colorimetric microdroplets printed on the surface of the CMOS imager ${ }^{24}$. However, to be compatible with modern electronics, a stable solid-state chemical sensing CMOS imager with multiplexed sensing capability is preferred, but not yet available. Substantial innovations are required for sensing materials coating, image processing, and sensing algorithms development.

In this work, we describe a method to turn a CMOS imager into an integrated solid-state chemical CMOS chip (C-CMOS) for simultaneous detection of multiple analytes (Fig. 1). To create different types of colorimetric sensing spots on the small and fragile imager surface, we sequentially spray droplets containing different sensing materials onto the surface of CMOS imager and then dry the droplets to form solid sensing spots. Comparing to conventional inkjet printing, the spray method enables depositing droplets with sizes much closer to a single pixel. Because the sensitivity is spot-size invariant (evidenced by experimental data) and each sensing spot is addressable through image processing, the spray method provides a simple and cost-effective approach for large-scale fabrication of C-CMOS chips. Though solid-state sensing elements are preferred because of their mechanical and chemical stability, achieving high sensitivity on the CMOS imager surface is challenging. This is because the small surface area of the sensing spot limits the number of active sensing sites, and the short optical path through the tiny sensing spot results in small optical absorbance change. We have solved these issues by introducing nanoparticles to the droplets, which enhances colorimetric sensing signals by several orders of magnitude. We have built integrated C-CMOS chips, tested their analytical performance, and demonstrated their compatibility with mobile electronics in realistic application scenarios by building and testing a C-CMOS chip-based smartphone accessory.

\section{Results}

\section{Fabrication of C-CMOS}

We transformed a conventional CMOS imager into a colorimetric sensor chip by directly spraying liquid microdroplets, each containing a sensing material in a solvent, onto the CMOS imager surface (Fig. 1e). Upon evaporation of the solvent, micron-scale solid colorimetric sensing spots were formed on the CMOS imager surface. The positions of the sensing spots were recorded by the CMOS imager during the spray process, allowing addressable labeling of each sensing spot. By sequentially spraying droplets of different sensing materials, sensing spots targeting different analytes were fabricated (Fig. 1b). Although printing fabrication provides a higher efficiency of pixel usage, we chose spray here because its capability to produce smaller droplets is desired for demonstrating the scalability of C-CMOS. Plus, it is a simple, efficient, and cost-effective way for large-scale fabrication of C-CMOS chips. The sensing spots are in direct contact with the CMOS imager surface, such that they can be clearly resolved without additional optical components, such as lens (Fig. 1f). 
Moreover, the C-CMOS sensor is much more size-effective than conventional colorimetric sensor because no focal distance is needed (Fig. 1d), making it more compatible with mobile electronics. An interesting finding is that the colorimetric sensing spots on the CMOS imager surface take the shape of a square with round corners (Figs. 1c, g and $\mathrm{h}$ ). This is due to the periodically arranged micro-lenses on the CMOS imager ${ }^{25}$ (Fig. 1c). By tracking the color changes of the sensing spots (Figs. $1 \mathrm{~g}$ and $\mathrm{h}$ ), different chemicals can be detected and quantified.

\section{Sensitivity enhancement with nanoparticles}

We fabricated C-CMOS chips for simultaneously sensing nitrogen dioxide $\left(\mathrm{NO}_{2}\right)$, carbon dioxide $\left(\mathrm{CO}_{2}\right)$, ammonia $\left(\mathrm{NH}_{3}\right)$, and acetone $\left(\mathrm{C}_{3} \mathrm{H}_{6} \mathrm{O}\right)$. These gases are either common air pollutants or important biomarkers ${ }^{26-29}$. Simply spraying droplets of sensing materials on the CMOS imager did not lead to acceptable sensitivity (black lines, Figs. 2a and b). This was because of the low surface-to-volume ratio and the short optical path of the tiny sensing spot (Figs. $2 \mathrm{c}$ and d). To enhance the sensitivity, we introduced silica nanoparticles $(\sim 10 \mathrm{~nm})$ into the sensing solutions, which drastically increased the surface-to-volume ratio (Figs. 2c and e). In addition, light scattering effect of the nanoparticles can prolong the optical path through the matrix of the sensing spot and increase optical absorbance change $\mathrm{j}^{30,31}$ (Fig. 2c). Consequently, the sensitivity increased by several orders of magnitude (Figs. 2a and b). However, if the concentration of silica nanoparticles was too high, the sprayed droplets tended to dry out quickly before reaching the CMOS imager surface, resulting in loose attachment and unstable optical signal.

\section{Calibration and detection limits}

We performed calibration on four types of sensing spots by measuring optical absorbance response to various concentrations of corresponding chemicals with 15 seconds exposure time (Fig. 3). Compared to detection tubes, which are semiquantitative and require minutes of sampling time, these micron-scale sensing spots show faster response time and more quantitative detection of chemical analytes (Table S1). According to our calculation, the detection limits are $0.16 \mathrm{ppm}, 71 \mathrm{ppm}, 0.33 \mathrm{ppm}$, and $445 \mathrm{ppm}$ for $\mathrm{NO}_{2}, \mathrm{CO}_{2}, \mathrm{NH}_{3}$ and $\mathrm{C}_{3} \mathrm{H}_{6} \mathrm{O}$, respectively.

\section{Scalability of sensitivity and noise}

We studied the sensitivity and noise dependence on the size of the sensing spot. The sensitivity is size invariant, as expected for an intensive quantity, color (Fig. 4a). This allows packing of increasing number of sensing spots on the same chip by decreasing the size of each sensing spot. This also makes the spray method practically effective for C-CMOS sensor fabrication, without worrying the distribution of sensing spots sizes affecting the sensitivity. The noise shows size dependence (Figs. 4b). Detail noise analysis (supplementary materials) indicates that read noise, dark noise and shot noise dominate. The first two are due to the CMOS imager and electronics, which can be improved through chip design. The shot noise is due to the finite number of photons and can be reduced by increasing incident light intensity and full well capacity of each pixel. All these types of noise are random and can be reduced by performing digital averaging either over multiple pixels or multiple frames (Fig. 4b). Benefiting from the spray method, we created and tested the smallest colorimetric sensing spot reported by far (Figs. 4c and d). Although as small as $\sim 10 \mu \mathrm{m}$ in diameter, its color change was clearly captured by the C-CMOS sensor. Its signal-tonoise ratio was $\sim 46$ after 3 min of exposure to $50 \mathrm{ppm} \mathrm{NH}$. This clearly demonstrated the excellent scalability of C-CMOS as a chemical sensing platform.

\section{Multiplex sensing of chemical analytes}

To demonstrate simultaneous detection of multiple analytes, we deposited all the four types of sensing spots on the same C-CMOS chip (Fig. 1f). Air from a gas cylinder was first flown over the C-CMOS for 5 min to obtain a stable baseline for each sensor, followed by introducing $3 \mathrm{ppm} \mathrm{NO}_{2}, 0.21 \% \mathrm{C}_{3} \mathrm{H}_{6} \mathrm{O}, 1.3 \%$ $\mathrm{CO}_{2}$, and $10 \mathrm{ppm} \mathrm{NH}$ sequentially, each lasted for $5 \mathrm{~min}$. After completing testing the four analytes, clean air was used to flush out residual chemicals. The optical absorbance changes upon exposure to each chemical (Fig. 5). It is noteworthy that $\mathrm{NH}_{3}$ induces a decrease in the optical absorbance of the $\mathrm{C}_{3} \mathrm{H}_{6} \mathrm{O}$ sensor. This is due to the reactions of $\mathrm{NH}_{3}$ with thymol blue on the $\mathrm{C}_{3} \mathrm{H}_{6} \mathrm{O}$ sensing spot. Although $\mathrm{NH}_{3}$ reacts with the $\mathrm{C}_{3} \mathrm{H}_{6} \mathrm{O}$ sensing spots, the $\mathrm{NH}_{3}$ sensing spots responds only to $\mathrm{NH}_{3}$, which can be combined with the responses of the $\mathrm{C}_{3} \mathrm{H}_{6} \mathrm{O}$ sensor for selective detection of $\mathrm{C}_{3} \mathrm{H}_{6} \mathrm{O}$. This example underscores the advantage of integrating multiple different sensing spots into a single C-CMOS chip for enhancing selectivity and reducing false detection. A similar strategy based on an array of cross-sensitive sensing elements has been widely used in electronic noses ${ }^{13,19}$. Although only four types of sensing spots are integrated here to illustrate the basic principle of C-CMOS chip, more sensing spots can be integrated for detection of more analytes, depending on the need of different applications.

\section{Integration with mobile electronics}

Besides the scalable and multiplexed chemical sensing capabilities, C-CMOS is intrinsically compatible to be integrated with mobile electronics. As a proof of concept, we built a C-CMOS chip-based smartphone accessary in the size of a USB flash drive for environmental monitoring and biomarker detection (Fig. 6a). When plugged in, the accessary can be powered by the smartphone (Samsung S8) and the images captured by the C-CMOS can be transmitted to the phone through a USB Type-C port for data processing. Indoor airborne transmission is a prominent way of SARS-CoV-2 infection in the ongoing pandemic of COVID19 , and $\mathrm{CO}_{2}$, co-exhaled with aerosols that may contain coronavirus, has been suggested as an indicator of social distance and infection risk ${ }^{32,33}$. Good ventilation that keeps $\mathrm{CO}_{2}$ lower than 550 ppm could make indoor air almost as virus-dispersing as outdoor air, reducing the risk of COVID-19 transmission ${ }^{32}$. We put the C-CMOS accessary in a room with the size of $4.2 \times 3.3 \times 2.7 \mathrm{~m}^{3}$, and the $\mathrm{CO}_{2}$ levels reported by the C-CMOS accessary changed with different ventilation and occupancy conditions (Fig. 6b), suggesting that it could be a useful personal tool for monitoring indoor infection risk. The accessary can also be used to detect biomarkers in biological samples. Abnormal urine ammonium $\left(\mathrm{NH}_{4}{ }^{+}\right)$levels are most often caused by kidney and liver diseases ${ }^{34}$, and thus monitoring urine $\mathrm{NH}_{4}{ }^{+}$could enable early diagnosis and management of diseases. $\mathrm{NH}_{4}{ }^{+}$ions in liquid are volatile and rapidly turn into $\mathrm{NH}_{3}$. We put the $\mathrm{C}$ - 
CMOS accessary on top of urine samples for 2 min to detect headspace $\mathrm{NH}_{3}$. The different responses to control and spiked samples clearly demonstrated the capability of the C-CMOS accessary as personal tool to detect volatile biomarkers (Fig. 6c). The compact format and easy connectivity with smart devices make the C-CMOS accessary a convenient tool for personal health management.

\section{Discussion}

We have demonstrated that a CMOS imager can be turned into an integrated chemical sensing chip (C-CMOS). The chip can perform colorimetric sensing of multiple chemicals without any additional optical components. This is enabled by depositing microdroplets containing various sensing materials on the surface of a CMOS imager chip to form colorimetric sensing spots. Each colorimetric sensing spot is able to provide quantitative and fast detection of chemical analyte. The performance is further enabled by implementing nanoparticles in the colorimetric sensing spots, allowing sensitive detection of analytes with sub-ppm level. Attributing to the convenient spray method, we have fabricated the smallest colorimetric gas sensing element reported by far, which is able to detect chemicals with high performance on the C-CMOS chip. We also show that the sensitivity is invariant of the sensing spot size, allowing further shrinking of sensing element size and future scaling up of the C-CMOS chip by packing increasing sensing spots on the chip.

Although spray method was used in this work to study the scalability and sensing performance of C-CMOS, high-throughput fabrication approaches with better control of droplet size and position, such as piezoelectric printer, may further increase the density of sensing spots and the efficiency of pixel usage. Thus, C-CMOS is also a promising platform for optoelectronic noses with large-scale sensor arrays. In addition, since some CMOS imagers already cost less than a dollar, the C-CMOS can even be designed as disposable sensor coated with reversible and irreversible colorimetric sensing materials, which further expands its application scenarios. In conclusion, C-CMOS is compact, multiplexed, low-cost, and compatible with existing microelectronics, which makes it an ideal chemical sensing unit for mobile and consumer electronics, robots, Internet-of-Things (loTs), electronic nose (eNose), and mHealth.

\section{Materials And Methods}

Materials. N,N-Dimethyl-1-naphthylamine, $m$-cresol purple, bromophenol blue, hydroxylamine sulfate, thymol blue, ethanol, $\mathrm{C}_{3} \mathrm{H}_{6} \mathrm{O}, \mathrm{glycerol}$, and silica nanoparticles ( $10 \mathrm{~nm}$ ) were purchased from Sigma-Aldrich and used as received. Ultra-pure water (18M) was produced by an ELGA Purelab Ultra RO system. $25 \mathrm{ppm} \mathrm{NO}, 100 \mathrm{ppm} \mathrm{NH}$, and 1.3\% $\mathrm{C}_{3} \mathrm{H}_{6} \mathrm{O}$ calibration gases were purchased from Cal Gas Direct, Inc. Air and $100 \% \mathrm{CO}_{2}$ were purchased from ASU Materials Management. Gas samples were prepared by diluting the high concentration calibration gases with clean air. By controlling the dilution ratio, gas samples with different concentrations of analytes were prepared.

Colorimetric sensing solutions. A mixture of water and ethanol (1:1 in volume) was used as solvent for all sensing solutions. Liquid N,N-Dimethyl-1naphthylamine was diluted to $1 \%(\mathrm{v} / \mathrm{v})$ for detection of $\mathrm{NO}_{2}$. $\mathrm{m}$-cresol purple $(1.1 \mathrm{mg} / \mathrm{mL})$ was used as $\mathrm{CO}_{2}$ sensing material. Bromophenol blue $(4 \mathrm{mg} / \mathrm{mL})$ was used for detection of $\mathrm{NH}_{3}$. Solution with dissolved hydroxylamine sulfate $(10 \mathrm{mg} / \mathrm{mL})$ and thymol blue $(0.6 \mathrm{mg} / \mathrm{mL})$ was mixed with glycerol $(1.5: 1 \mathrm{in}$ volume) and used for $\mathrm{C}_{3} \mathrm{H}_{6} \mathrm{O}$ detection.

Fabrication of C-CMOS. The sensing solutions were injected into $3 \mathrm{~mL}$ manually operated polypropylene spray bottles and mixed for 1 minute using a vortex mixer before spray fabrication. A 5-megapixel CMOS imager (ov5647, OmniVision Technologies) with $1.4 \mu \mathrm{m}$ pixel size and $3.67 \mathrm{~mm} \times 2.74 \mathrm{~mm}$ imaging area was used. The lens and filter of the CMOS imager were removed so that the microdroplets can be directly coated on the microlenses of the CMOS imager. To coat the microdroplets, one sprayer is fixed on a retort stand and placed vertically $50 \mathrm{~cm}$ above the CMOS imager (accurate alignment is not needed). After spraying one sensing solution, the sprayer was replaced by another one to spray another type of sensing solution.

Experimental setup. A customized polypropylene chamber ( $0.5 \mathrm{~mL}$ inner volume) was fixed on the C-CMOS for gas sample delivery. A white LED (LEDtronics) was used as a light source. A small diaphragm gas pump (0.8 L/min, Topsflo) was used to deliver gas samples through the chamber. The CCMOS was connected to a small single-board computer (Raspberry Pi 3B) for configuration and image recording. Gain and white balance of the CMOS imager were adjusted so that the captured images were bright but not saturated, and the flat-field corrected images showed a neutral gray color (Fig. S1). Full resolution images $(2592 \times 1944)$ were captured with a frame rate of $\sim 88 \mathrm{frames} / \mathrm{min}$.

Data processing and calibration. To minimize the variation in pixel to pixel light sensitivity and dark currents, the CMOS imager was calibrated before spraying so that the captured images during experiments can be flat-field corrected ${ }^{35}$ (Fig. S1). The procedure of obtaining absorbance signals of the sensing elements is shown in Fig. S2. Captured images were flat-field corrected first. Then ImageJ was used to identify the sensing elements and measure the intensities of these sensing elements $\left(I_{\text {sensor }}\right)$ and a blank reference area $\left(I_{\text {ref }}\right)$. Absorbance $\left(-\log \left(I_{\text {sensor }} / I_{\text {ref }}\right)\right)$ change as a function of time for each sensing element was calculated, and this signal was smoothed using a smoothing window of 22 frames (15 s). Signals of different color channels were used for different types of sensors for better signal to noise ratio, i.e. red channel for $\mathrm{NH}_{3}$ sensing elements, green channel for $\mathrm{C}_{3} \mathrm{H}_{6} \mathrm{O}$ sensing elements, blue channel for $\mathrm{NO}_{2}$ sensing elements, and gray channel (average of RGB channels) for $\mathrm{CO}_{2}$ sensing elements.

To improve the performance of $\mathrm{NO}_{2}$ and $\mathrm{CO}_{2}$ detection, $66.7 \mathrm{mg}$ silica nanoparticles were added into $1 \mathrm{~mL} \mathrm{NO}_{2}$ and $\mathrm{CO}_{2}$ sensing solutions, respectively. $\mathrm{No}$ silica nanoparticles were added into $\mathrm{NH}_{3}$ and $\mathrm{C}_{3} \mathrm{H}_{6} \mathrm{O}$ sensing solutions. In the calibration of $\mathrm{NO}_{2}$ sensing elements, before delivering each $\mathrm{NO}_{2}$ gas sample, 1 ppm NO$N_{2}$ was delivered for $1 \mathrm{~min}$ and the absorbance change $\left(\triangle A b s_{1 \mathrm{ppm}}\right)$ was recorded as an internal reference signal. Then the $\mathrm{NO}_{2}$ gas sample was delivered and the absorbance change of the first $15 \mathrm{~s}\left(\triangle A b s_{15 \mathrm{~s}}\right)$ was normalized $\left(\triangle A b s_{15 \mathrm{~s}} / \triangle A b s_{1} \mathrm{ppm}\right)$ as the result used in the calibration. In the calibration of $\mathrm{NH}_{3}$ sensing elements, the initial absorbance value $\left(A b s_{\text {init }}\right)$ of each sensing element was recorded as an internal reference signal. After $15 \mathrm{~s}$ of $\mathrm{NH}_{3}$ sampling, absorbance change $\left(\triangle A b s_{15 s}\right)$ was normalized $\left(\triangle A b s_{15 s} / A b s_{\text {init }}\right)$ as the result used in the calibration. In the calibration of $\mathrm{C}_{3} \mathrm{H}_{6} \mathrm{O}$ sensing elements, after $15 \mathrm{~s}$ 
of $\mathrm{C}_{3} \mathrm{H}_{6} \mathrm{O}$ sampling, the absorbance change $\left(\Delta A b s_{15 s}\right)$ was used as the signal. Because $\mathrm{CO}_{2}$ sensing elements are fully reversible, each element can be tested repeatedly and calibrated individually. Figure $4 \mathrm{~B}$ shows the calibration of one $\mathrm{CO}_{2}$ sensing element, and each concentration was tested three times.

Fabrication of C-CMOS smartphone accessary. A homemade circuit board was used to connect a C-CMOS chip to a smartphone (Samsung S8) through a USB Type-C port and USB video class (UVC) protocol. A white LED (LEDtronics) was used as a light source. A black housing with gas diffusion ports was 3D printed to block ambient light.

\section{Declarations}

\section{Competing interests:}

The authors declare no competing interests.

\section{Author contributions:}

N.T., D.W., X.X., and C.J. conceived project. D.W. and F.Z. performed experiments and data analysis. V.V.T. helped with programming. F.Z., K.R.M., J.Y., and E.F. helped with the optimization of sensing recipes. N.T., D.W., F.Z., C.J., and X.X. wrote the manuscript. All authors reviewed and commented on the manuscript.

\section{Acknowledgments}

This work was supported by Major Scientific Project of Zhejiang Lab (Grant No.2020MC0AD01).

\section{References}

1. Yu, K. H., Beam, A. L. \& Kohane, I. S. Artificial intelligence in healthcare. Nature Biomedical Engineering 2, $719-731$ (2018).

2. Topol, E. J. High-performance medicine: the convergence of human and artificial intelligence. Nature Medicine 25, 44-56 (2019).

3. Cully, A., Clune, J., Tarapore, D. \& Mouret, J.B. Robots that can adapt like animals. Nature 521, 503 (2015).

4. Tao, N. Adding new senses to your cell phone: opportunities for gas sensing. ACS Sensors 2, 317 (2017).

5. Perkel, J. M. Pocket laboratories. Nature 545, 119-122 (2017).

6. Rappaport, S. M. \& Smith, M. T. Environment and disease risks. Science 330, 460-461 (2010).

7. Hakim, M. et al. Volatile organic compounds of lung cancer and possible biochemical pathways. Chemical Reviews 112, 5949-5966 (2012).

8. Ellis, D. I. et al. Fingerprinting food: current technologies for the detection of food adulteration and contamination. Chemical Society Reviews 41, 57065727 (2012)

9. Tricoli, A., Nasiri, N. \& De, S. Wearable and miniaturized sensor technologies for personalized and preventive medicine. Advanced Functional Materials 27, 1605271 (2017).

10. Bandodkar, A. J., Jeerapan, I. \& Wang, J. Wearable chemical sensors: present challenges and future prospects. ACS Sensors 1, 464-482 (2016).

11. Korotcenkov, G. Metal oxides for solid-state gas sensors: What determines our choice? Materials Science and Engineering: B 139, 1-23 (2007).

12. Dey, A. Semiconductor metal oxide gas sensors: A review. Materials Science and Engineering: B 229, 206-217 (2018).

13. Li, Z., Askim, J. R. \& Suslick, K. S. The optoelectronic nose: colorimetric and fluorometric sensor arrays. Chemical Reviews 119, 231-292 (2018).

14. Borisov, S. M. \& Wolfbeis, O. S. Optical biosensors. Chemical Reviews 108, 423-461 (2008).

15. Seto, Y. et al. Sensing technology for chemical-warfare agents and its evaluation using authentic agents. Sensors and Actuators B: Chemical 108, 193197 (2005).

16. Bolstad-Johnson, D. M. et al. Characterization of firefighter exposures during fire overhaul. American Industrial Hygiene Association 61, 636-641 (2000).

17. Seto, Y. et al. Development of an on-site detection method for chemical and biological warfare agents. Toxin Reviews 26, 299-312 (2007).

18. Hunt, A. \& Alder, J. Quantitative analysis of chlorine in air by gas chromatography. Analytical Communications 33, 61-64 (1996).

19. LaGasse, M. K. et al. Colorimetric sensor arrays: development and application to art conservation. Journal of the American Institute for Conservation $\mathbf{5 7}$, 127-140 (2018).

20. Kim, S. Y., Li, J., Lim, N. R., Kang, B. S. \& Park, H. J. Prediction of warmed-over flavour development in cooked chicken by colorimetric sensor array. Food Chemistry 211, 440-447 (2016)

21. Shrestha, N. K. et al. The combined rapid detection and species-level identification of yeasts in simulated blood culture using a colorimetric sensor array. PloS one 12, e0173130 (2017).

22. Zhihua, L. et al. Characterization of colorimetric sensor arrays by a multi-spectral technique. Analytical Methods 8, 2357-2365 (2016).

23. Chung, S. et al. Colorimetric sensor array for white wine tasting. Sensors 15, 18197-18208 (2015).

24. Mallires, K. R., Wang, D., Wiktor, P. \& Tao, N. A Microdroplet-Based Colorimetric Sensing Platform on a CMOS Imager Chip. Analytical Chemistry 92, 93629369 (2020). 
25. Raj, R., Adera, S., Enright, R. \& Wang, E. N. High-resolution liquid patterns via three-dimensional droplet shape control. Nature Communications 5, 4975 (2014).

26. Landrigan, P. J. et al. The lancet commission on pollution and health. The Lancet 391, 462-512 (2018).

27. Kogevinas, M. et al. Exposure to substances in the workplace and new-onset asthma: an international prospective population-based study (ECRHS-II). The Lancet 370, 336-341 (2007).

28. Grandjean, P. \& Landrigan, P. J. Developmental neurotoxicity of industrial chemicals. The Lancet 368, 2167-2178 (2006).

29. Kirschbaum, B., Sica, D., Anderson, F. P. Urine electrolytes and the urine anion and osmolar gaps. Journal of Laboratory and Clinical Medicine 133, 597604 (1999).

30. Arakawa, H., Yamaguchi, T., Takeuchi, A. \& Agatsuma, S. Efficiency improvement of dye-sensitized solar cell by light confined effect. IEEE 4th World Conference on Photovoltaic Energy Conference. 36-39 (2006).

31. Derkacs, D. et al. Nanoparticle-induced light scattering for improved performance of quantum-well solar cells. Applied Physical Letters 93,091107 (2008).

32. Gilbert, R. Could carbon dioxide monitoring allow more indoor activity during the pandemic?

http://richardgilbert.ca/Files/2020/Could\%20carbon\%20dioxide\%20monitoring\%20allow\%20more\%20indoor\%20activity\%20during\%20the\%20pandemic\% (2020).

33. Peng, Z. \& Jimenez, J. L. Exhaled CO2 as COVID-19 infection risk proxy for different indoor environments and activities. Preprinted at https://doi.org/10.1101/2020.09.09.20191676 (2020).

34. Liu, N.Y. et al. A handheld, colorimetric optoelectronic dynamics analyzer for measuring total ammonia of biological samples. 6, 1-10 (2018).

35. Emerson, G. P. \& Little, S. J. Flat-fielding for CCDs in AAVSO observations, I. Journal of the American Association of Variable Star Observers 27, 49-54 (1999).

\section{Figures}

a

Conventional tubes

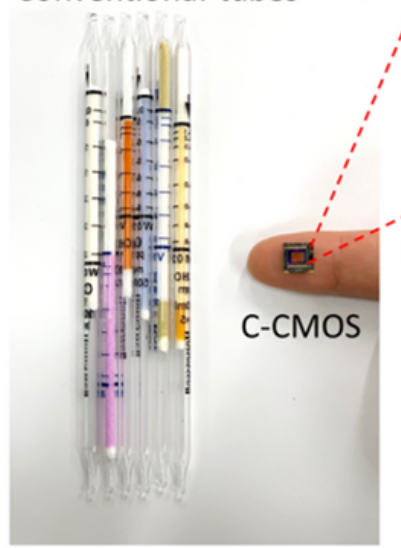

e

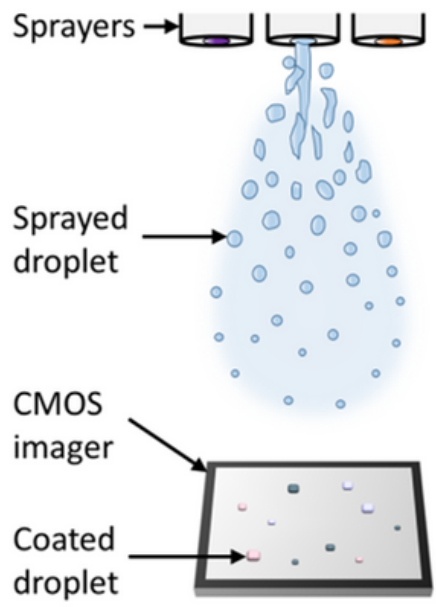

d

Conventional colorimetric sensor array

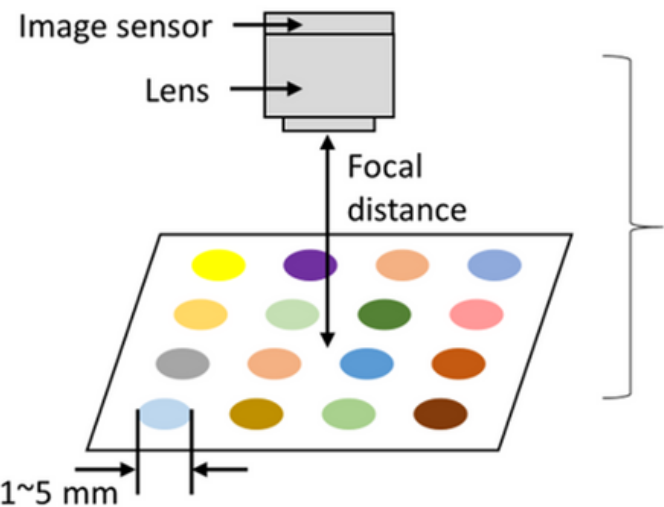

1 5 $\overrightarrow{\mathrm{mm}}$
C-CMOS

Image sensor

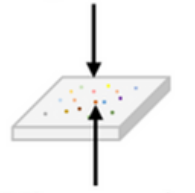

Micron-scale sensing spots

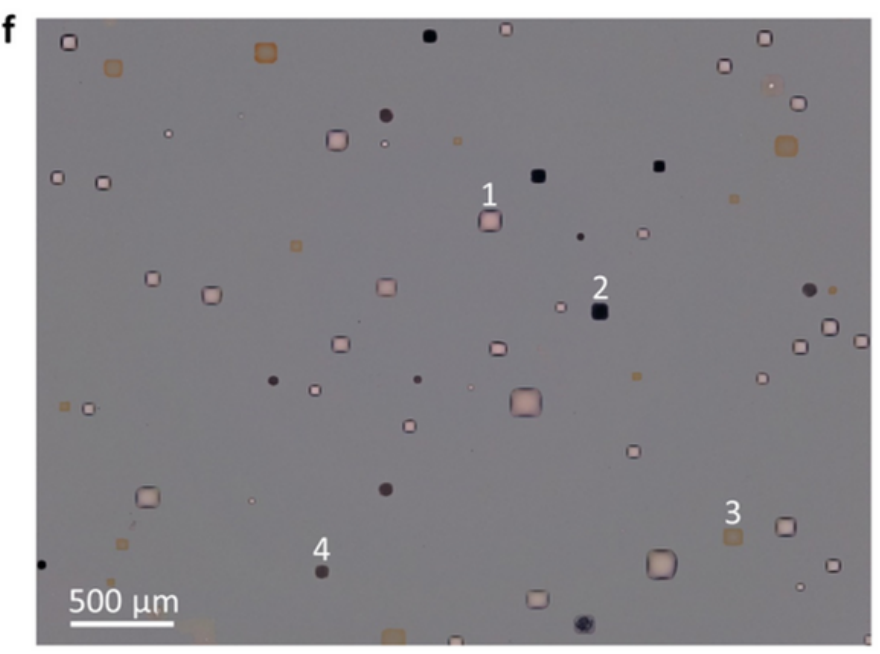

g

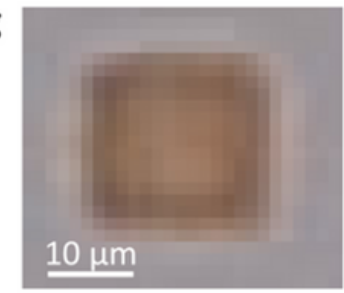

h

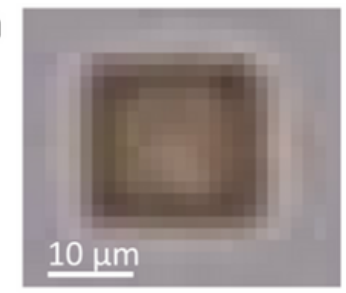

Figure 1 
Principle and fabrication of C-CMOS. a, Comparison of a C-CMOS with conventional colorimetric detection tubes. b, Optical microscopic image of a C-CMOS with multiple sensing spots, each functions like a detection tube. c, Scanning electron microscopic (SEM) image of a sensing spot. d, Schematic comparison of a C-CMOS with conventional colorimetric sensor array. e, Schematic of C-CMOS fabrication process. Microdroplets containing sensing materials and nanoparticles were sprayed onto a CMOS imager. By sequentially spraying microdroplets containing different sensing materials, a C-CMOS imager capable of detecting different chemicals was fabricated. f, Image of sensing spots recorded by a C-CMOS, where numbers, 1, 2, 3, 4, represent C3H6O, CO2, NH3, and NO2 sensing spots, respectively. $\mathrm{g}$ and $\mathrm{h}$ show an $\mathrm{NH} 3$ sensing spot before and after $\mathrm{NH} 3$ exposure.

a

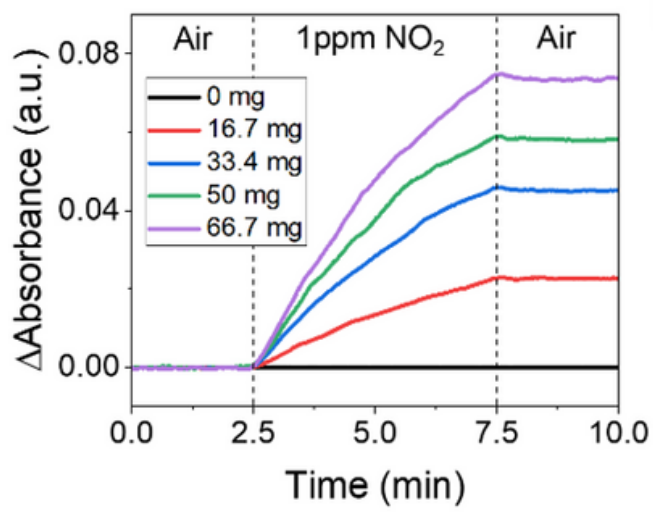

\section{d Dried droplet without nanoparticles}

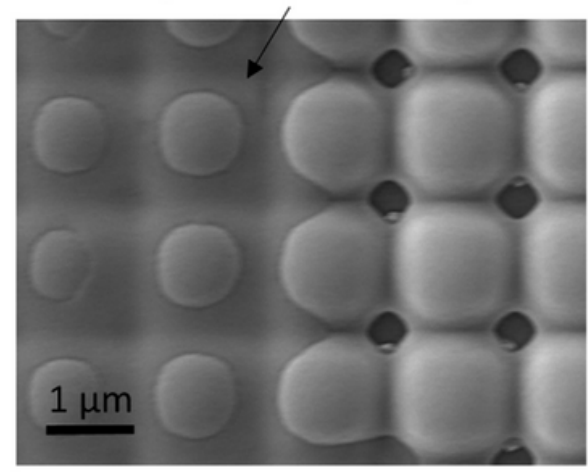

b

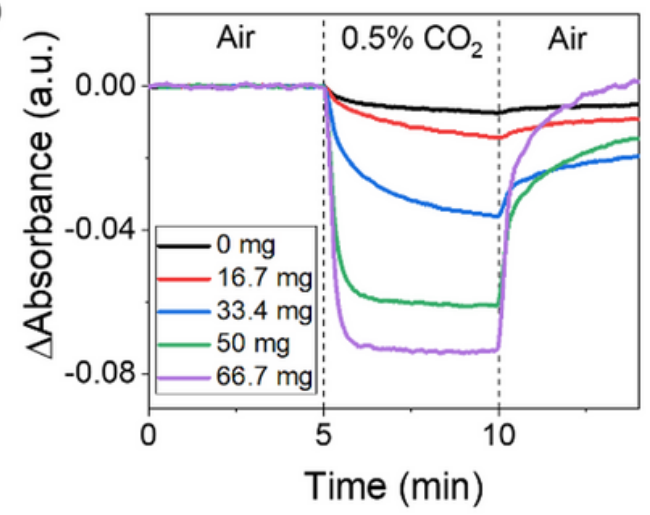

e Dried droplet with nanoparticles

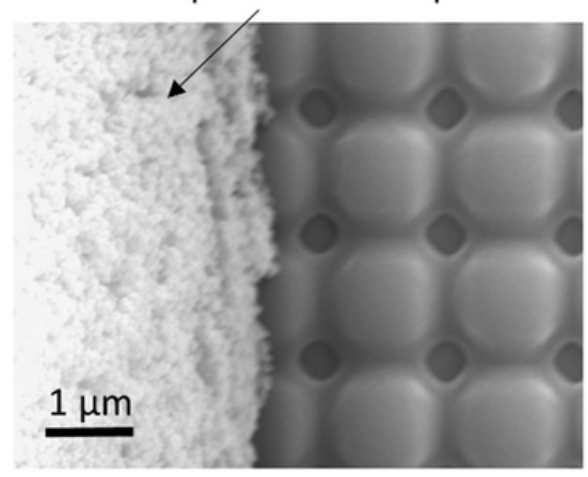

C

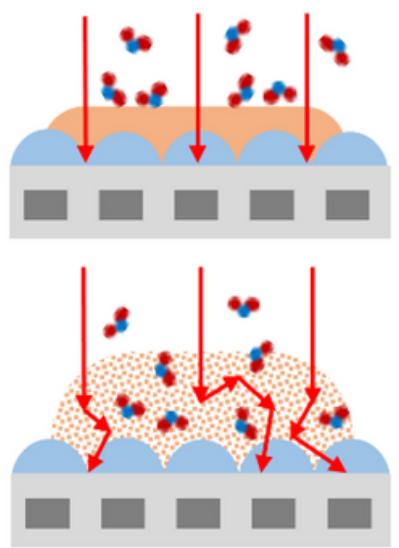

Sensing spot without nanoparticles

\section{Sensing spot with nanoparticles}

\section{Gas analyte}

$\longrightarrow$ Light path

\section{CMOS imager pixel}

Figure 2

Enhancing performance by including silica nanoparticles. a, Optical response to NO2 of NO2 sensing spots containing different amounts of silica nanoparticles in $1 \mathrm{~mL} \mathrm{NO} 2$ sensing solution, showing increasing sensitivity with increasing silica nanoparticle loading. b, Optical response to $\mathrm{CO} 2$ of $\mathrm{CO} 2$ sensing spots containing different amounts of silica nanoparticles in $1 \mathrm{~mL} \mathrm{CO2} \mathrm{sensing} \mathrm{solution.} \mathrm{Sensitivity,} \mathrm{response} \mathrm{time,} \mathrm{and} \mathrm{recovery} \mathrm{time} \mathrm{are} \mathrm{all}$ enhanced by the silica nanoparticles. c, lllustration of sensing spots with and without silica nanoparticles. d, SEM image of the edge of a NO2 sensing spot without silica nanoparticles. e, SEM image of the edge of a NO2 sensing spot including silica nanoparticles, showing a porous structure with high surface area, responsible for the sensitivity enhancement. 

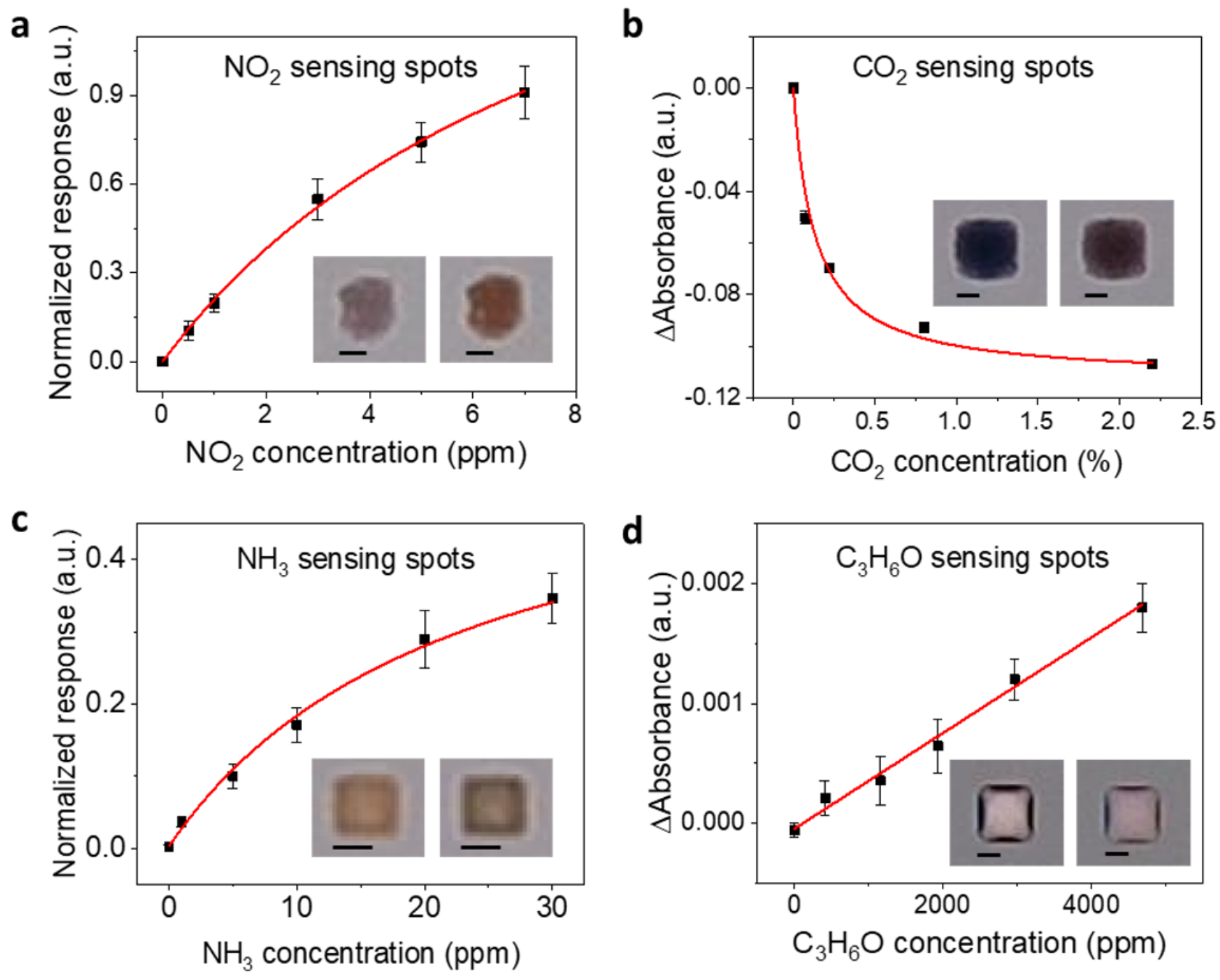

Figure 3

Calibrations of four types of colorimetric sensing spots on CMOS imager. a, NO2 sensing spots. b, A CO2 sensing spots. c, NH3 sensing spots. d, C3H6O sensing spots. The error bars in the calibration plots of $\mathrm{NO} 2, \mathrm{NH} 3$, and $\mathrm{C} 3 \mathrm{H} 6 \mathrm{O}$ sensing spots represent the standard deviation obtained from different sensing spots $(n>5)$. The error bars for the $\mathrm{CO} 2$ sensing spots are smaller than the size of the data points. The red lines represent the fitting curves (Langmuir model for $\mathrm{NO} 2, \mathrm{CO} 2$, and $\mathrm{NH} 3$, and linear fitting for $\mathrm{C} 3 \mathrm{H} 6 \mathrm{O}$ ). The inset photos captured by the $\mathrm{C}$-CMOS chip show the colors of each sensing spot before (left) and after (right) exposure to the chemical analyte, where the scale bars represent 10 pixels $(14 \mu \mathrm{m})$. 


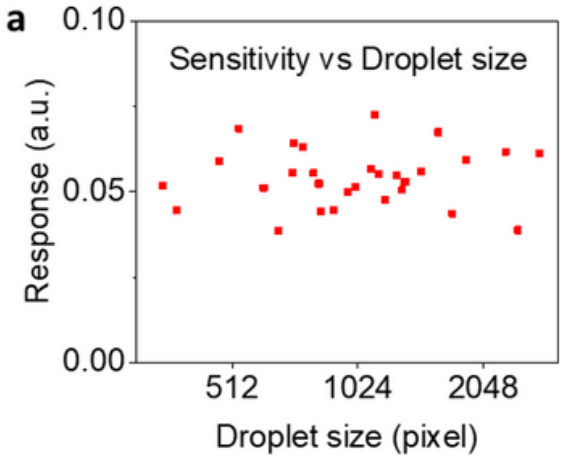

d

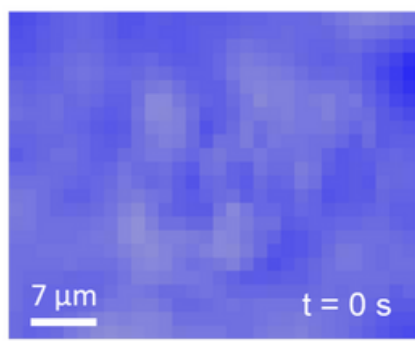

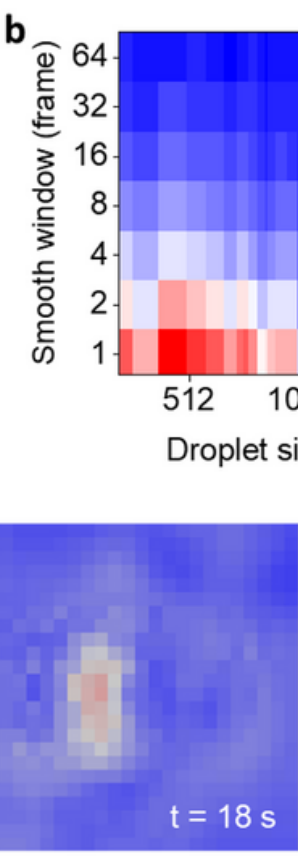
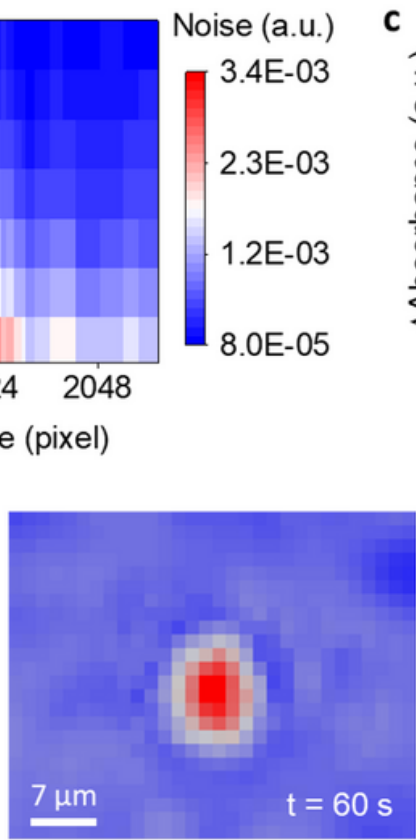
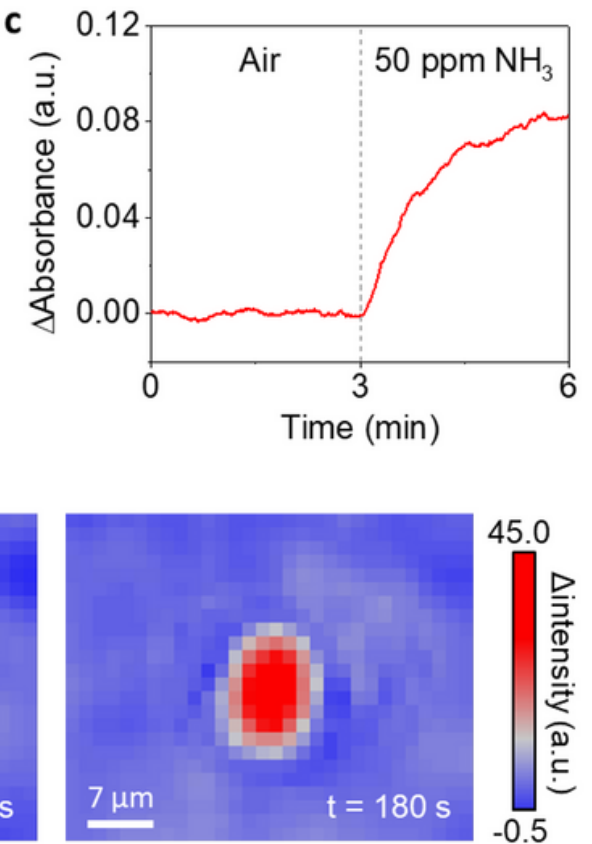

\section{Figure 4}

Scalability of sensitivity and noise. a, The sensitivity of NO2 sensing spots and the size of sensing spots show no correlation. The sensitivity is measured as the response to $1 \mathrm{ppm} \mathrm{NO2} \mathrm{for} 1 \mathrm{~min}$. b, Dependence of absorbance noise (standard deviation) on sensing spot size and frame number averaged over, showing decreasing noise with increasing sensing spot size and frame number. c, Response of the smallest sensing spot ( $10 \mu \mathrm{m}$ in diameter) to 50 ppm NH3. d, Differential images showing the intensity change of the smallest sensing spot during exposure to 50 ppm NH3.

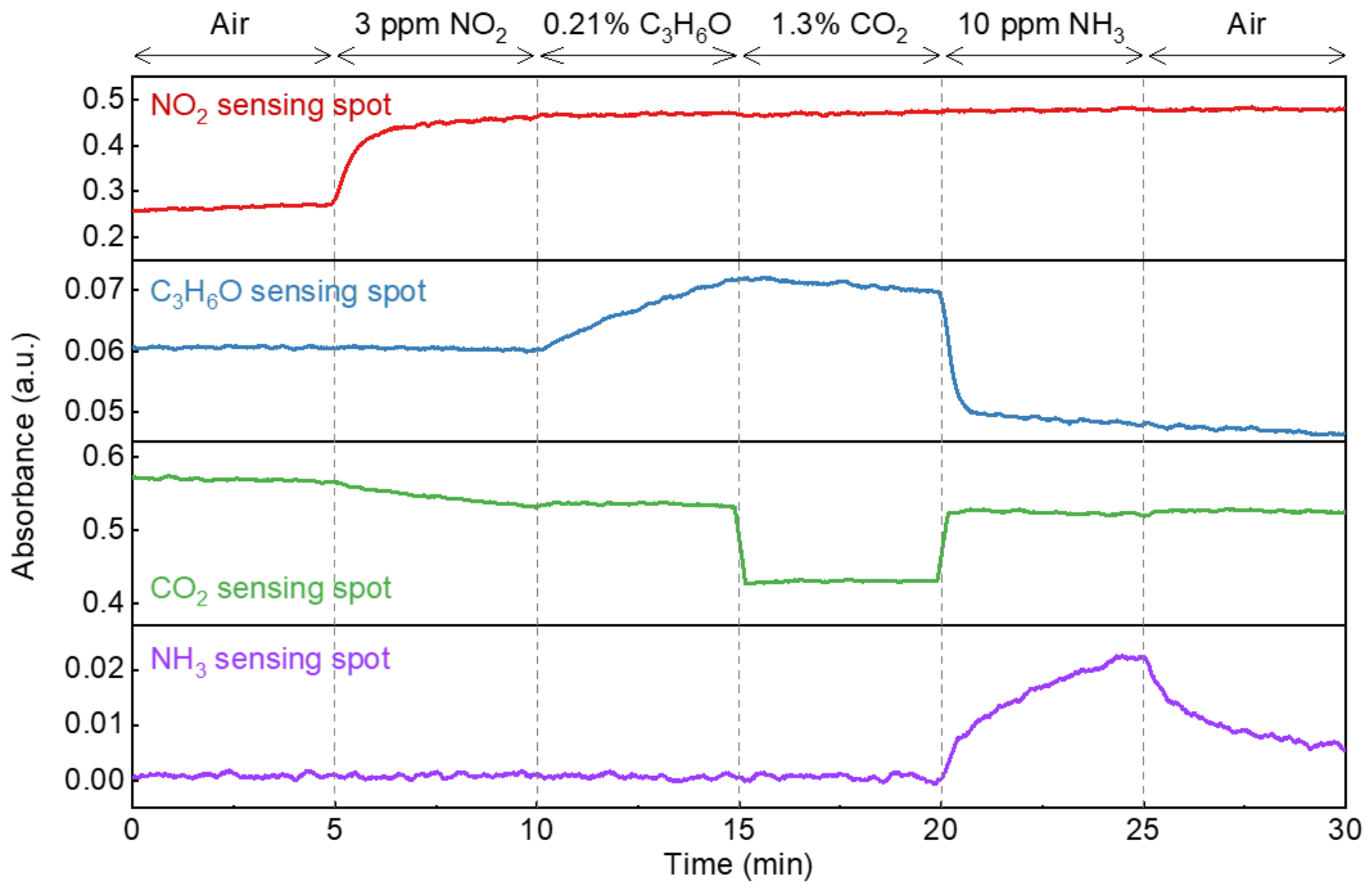

Figure 5 
Multiplexed chemical detection. Air was delivered for 5 minutes in the beginning and end of the test. In between, 3 ppm NO2, $0.21 \% \mathrm{C} 3 \mathrm{H} 60,1.3 \% \mathrm{CO} 2,10 \mathrm{ppm}$ $\mathrm{NH} 3$, were sequentially delivered, and each lasted for 5 minutes. The data were collected using the C-CMOS shown in Fig. $1 \mathrm{f}$.

a

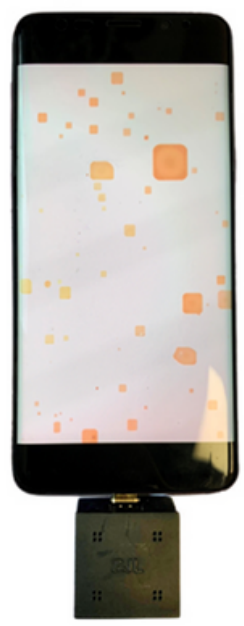

b

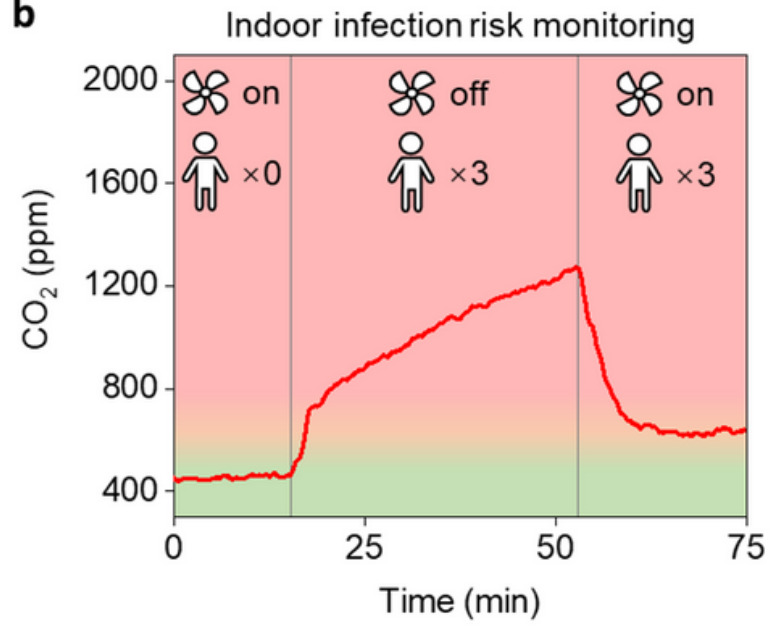

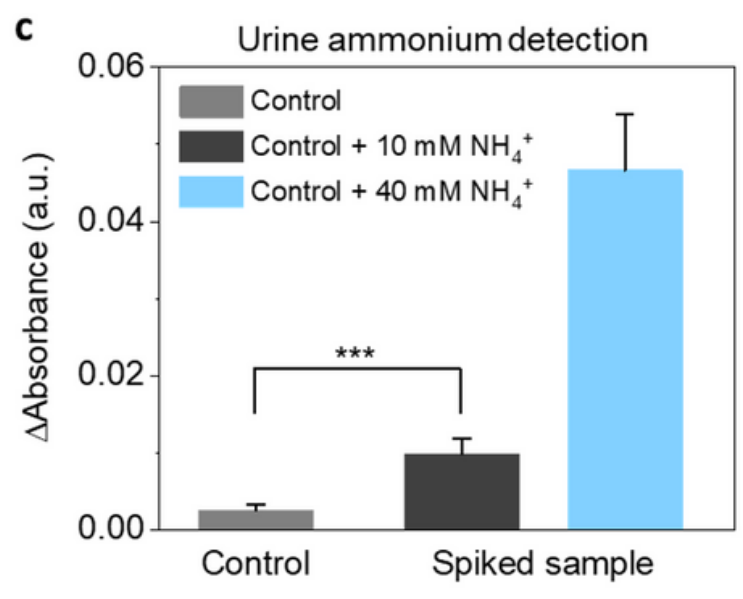

Figure 6

Integration with mobile electronics and example applications. a, A photo of a smartphone accessary with a built-in C-CMOS chip connected to a phone. b, Measuring indoor $\mathrm{CO} 2$ as an indicator of indoor infection risk. $\mathrm{CO} 2$ levels of a $\sim 37 \mathrm{~m} 3$ room recorded by the C-CMOS accessary change with different ventilation and occupancy conditions. c, Urine ammonia detection with the C-CMOS accessary. The urine sample of a health subject was divided into three parts, two of which were spiked with different amount of ammonium hydroxide. The error bars represent the standard deviation obtained from 5 different sensing elements. ${ }^{* \star} p<0.001$.

\section{Supplementary Files}

This is a list of supplementary files associated with this preprint. Click to download.

- Supplementaryinformation.pdf 